Franjić $S^{1^{*}}$

\title{
In Shortly about Cancer of the Tongue
}

${ }^{1}$ Faculty of Law, International University of Brcko District, Brcko, Bosnia and Herzegovina

Corresponding Author: Siniša Franjić

Address: Faculty of Law, International University of Brcko District, Brcko, Bosnia and Herzegovina; Tel: +387-49-4904-6o; E-mail: sinisa.franjic@gmail.com

Received date: 02 February 2020; Accepted date: 07 March 2020; Published date: 14 March 2020

Citation: Franjić S. In Shortly about Cancer of the Tongue. J Health Care and Research. 2020 Mar 07;1(1):7-11.

Copyright (C) 2020 Franjić S. This is an open-access article distributed under the Creative Commons Attribution License, which permits unrestricted use, distribution, and reproduction in any medium, provided the original work is properly cited.

\section{Abstract}

Cancer of the tongue is a type of oral cancer that occurs in the front two-thirds of the tongue. If cancer occurs at the back of the tongue, then it is a type of head or neck cancer. These cancers are extremely rare and occur in a small number of cases. Cancer in the front of the tongue also does not occur as often, but it is the most common type of oral cancer. Cancer of the tongue mainly occurs in squamous cells - thin, flat cells that are found on the surface of all soft tissues, and therefore the tongue. Cancer of the tongue is a disease that affects the front of the tongue, while cancer in the back of the tongue is called oropharyngeal cancer. Symptoms that indicate tongue cancer are pain in the jaw or throat, pain during swallowing, feeling that there is something in the throat, stiff tongue or jaw, problem with chewing and swallowing food, white or red stamps in the mouth or tongue, ulcer on non-healing tongue, pimples in the mouth, bleeding tongue and growths on the tongue.
\end{abstract}

\section{Keywords}

Tongue; Cancer; Diagnosis; Health

\section{Introduction}

An epulis is a granulomatous lesion of the gingiva [1]. It represents an exaggerated inflammatory response to a minor injury. Only symptomatic epulides need to be excised. Peripheral giant cell reparative granulomas also occur commonly on the gingiva. The "giant" cell of origin appears to resemble an osteoclast. These granulomas are polypoid, submucosal, and fibrous. The radiographic examination may reveal the erosion of the underlying bone. Excision must be complete to prevent a recurrence.

The tongue and larynx are common locations for the development of papillomas. They are caused by the human papillomavirus, which induces squamous epithelial proliferation. Eradication may be accomplished by excision or cauterization.

Granular cell myoblastoma is a rare benign tumor of the tongue that originally was described as of embryonal muscle cell origin. These tumors are now believed to derive from Schwann cells and have been found to arise throughout the aerodigestive tract. In the tongue, these tumors form firm submucosal swellings in the middle third and can mimic squamous cell carcinoma. Wedge excision is recommended.

Ulcers of the oral lining are common. The idiopathic aphthous ulcer is the most common type. The cycle of painful ulceration and spontaneous healing may occur several times a year. Viral infections, nutritional deficiencies, and emotional stress are common etiologic factors. These ulcers often respond to topical 
steroids.

Lichen planus is a degenerative mucocutaneous disease with a probable autoimmune basis. The oral lesions appear with or without cutaneous manifestations and may, at times, become erosive. Squamous cell carcinoma has been found in association with lichen planus infection. Systemic and topical retinoids are being evaluated in the treatment of this condition.

\section{Cancer}

Cancer of the oral tongue is second only to the lip as the most common primary site [1]. Tobacco and alcohol are the most common associated conditions. Immunosuppressed patients of any age, however, may be at higher risk for tongue cancer. The tongue is a complex muscular structure receiving motor intervention from the hypoglossal nerve.

The tongue is connected to the hyoid bone by the hyoglossus muscle and superiorly to the mandible by the genioglossus muscle. The ventral surface of the tongue has openings of the sublingual ducts. On the dorsal surface are papillae with specialized sensory organs for taste. Malignancy of the tongue occurs most frequently at the midportion of the lateral tongue and is often asymptomatic. Radial spread through the tongue may extend submucosally to the base of the tongue and across the midline or laterally to the floor of the mouth. Ipsilateral metastases are common to the submandibular and submental nodes. Clinical evidence of cervical metastasis is present in 40-60 percent of patients. Survival rates are 70-90 percent for localized disease and 30-40 percent for regional metastases.

Definitive therapy for carcinoma of the tongue can be attempted with both external-beam radiation and interstitial radiation. External radiation in doses of 6500 cGy may be useful, but implantation of afterloading devices can deliver doses in the range of 10,000-15,000 cGy over a small area with greater effect.

The surgical therapy of carcinoma of the tongue consists of resection of the tumor with a margin of normal tissue and en bloc removal of the regional lymph nodes. Most surgeons are uneasy about the ability to obtain clear margins with such resections, which has led to the common use of adjuvant radiation therapy. Resection at the base of the tongue may predispose the patient to aspiration and ultimately respiratory failure. Despite these problems, total glossectomy with or without laryngectomy is a valuable procedure for both cure and palliation. The 3year survival of 53 percent has been achieved in one series, with 80 percent of patients demonstrating intelligible speech if the larynx is preserved and 93 percent regaining the ability to maintain their nutritional status by oral alimentation.

There is no satisfactory way to reconstruct the tongue. Denervation of the tongue by resection or injury to both hypoglossal nerves usually renders the patient incapable of swallowing or effective speech. After surgical resection of a portion of the tongue, the reconstructive goal is to allow free mobility of the remaining tongue while providing a watertight seal to the oral cavity. Advancing the posterior mobile tongue or setting back the excess anterior tongue may provide the optimal solution. The defect of total glossectomy involves the tongue, floor of the mouth, and sometimes the pharyngeal and laryngeal mucosa. Restoration of oral continence usually requires significant soft tissue. The pectoralis major flap serves well to replace the entire floor of the mouth, as does the jejunal free flap, which also can replace the pharynx and crevice esophagus. When a portion of the mandible must be resected for carcinoma of the oral tongue, the urgency of reconstruction depends on what part of the mandible has been resected. Resection of the symphysis or anterior segment of the mandible is a devastating problem that requires immediate reconstruction. Vascularized bone from the scapula, fibula, iliac crest, radius, or metatarsal is an excellent method of reconstruction. Other areas of the mandible are less demanding for reconstruction.

Cancers of the oral tongue often are associated with occult cervical lymph node metastases. Selective neck dissection is combined with primary resection (usually hemiglossectomy) in all but the most superficial 
lesions [2]. Forty percent to $70 \%$ of patients with cancers of the floor of the mouth larger than $2 \mathrm{~cm}$ have occult lymph node metastases. Because of this, surgical resection includes selective neck dissection or cervical lymph node irradiation. Early cancers of the retromolar trigone or alveolar ridge are treated effectively by transoral resection. More advanced lesions may require mandibulectomy and neck dissection, followed by postoperative radiation. Lesions of the palate and all abnormal-appearing lesions need a biopsy.

\section{Symptoms}

The patients are usually over the age of 50 years [3]. The peak incidence is between 60 and 70 years.

Males were affected far more than females when syphilis and pipe smoking were common. Now there are almost as many women affected as men.

The commonest complaint is of a painless lump or irregularity on the surface of the tongue. If the early lesion is ignored or not noticed, the patient may not present until they have an enlarging ulcer causing pain in the tongue (sometimes referred to the ear), excessive salivation, difficulty with mastication and swallowing, and fetor oris.

If the lesion has spread extensively before it is noticed, it may cause immobility of the tongue (ankyloglossia) and difficulty with speech. Lesions on the back of the tongue may alter the quality of the voice.

Alternatively, the patient may present with a lump in the neck (enlarged lymph glands) before they notice any abnormality in the tongue. The tumor itself tends to occur on the lateral border of the tongue, and rarely affects the dorsum [4].

Anterior tumors commence as a nodule, fissure or ulcer, although occasionally a widely infiltrating type of tumor is seen. At first, the lesion is painless but becomes painful as it invades and becomes grossly septic. The pain often radiates to the ear, being referred from the lingual branch of the trigeminal nerve, supplying the tongue, along its auriculotemporal branch. Ulceration is accompanied by bleeding. The typical picture of the late disease is an old man sitting in the outpatient department spitting blood into his handkerchief, with a plug of cotton wool in his ear. As the tumor extends onto the floor of the mouth and the alveolus, speech and swallowing become difficult because of the fixation of the tongue (ankyloglossia). Palpation is especially valuable. Malignant ulcers in the mouth, as in the rectum, feel hard with surrounding induration.

Lymphatic spread occurs to the submental, submandibular and deep cervical nodes. Unless the primary tumor is situated far laterally on the margin of the tongue, this lymphatic spread may be bilateral.

Posterior third tumors of the tongue are rapidly growing and are of the lymphoepithelioma type with early spread bilaterally to the cervical nodes.

Any nodule or chronic ulcer of the tongue must be regarded with great suspicion of malignant disease, particularly if there is any predisposing factor such as leucoplakia.

\section{Forms}

Carcinomata of the tongue may present in four forms: an ulcer, a nodule, a papilliferous or warty nodule, or a fissure in an area of induration [3].

A carcinomatous lump tends to be ovoid, with its long axis parallel to the mid-line of the tongue. It may vary in size from a small nodule to a mass $2-5 \mathrm{~cm}$ across. It feels hard and has an indistinct edge where it is spreading into the rest of the tongue.

A carcinomatous ulcer of the tongue usually has the typical features of carcinoma: a florid, friable, bleeding, everted edge, a sloughing yellow-grey base, a thin serous discharge and induration of the surrounding tissues.

The papilliferous or warty carcinoma looks like any other papilloma in that it is covered with an excess of proliferating filiform epithelium, which is usually paler 
than the surrounding pink epithelium, but the base is broad and firm and the area of the tongue from which it arises is indurated. It may be of any size but rarely juts out far from the tongue because of the restriction of the mouth.

The fissure in an area of carcinomatous induration is a rare form of tongue cancer. It is a modified form of the nodule which has spread so diffusely that it does not have a detectable edge. The fissure may be a cleft in the tongue that has deepened and lost its epithelium or a deep linear ulcer.

Carcinoma of the tongue originates most frequently in the middle third of the lateral border but other parts, including the posterior and upper surfaces, are occasionally implicated [5]. The earliest recognizable changes are those of erythroplakia and induration; they progress to ulceration. The ulcer is characteristically bounded by raised, firm margins. On palpation, the extent of the tumor is usually found to be greater than the appearances suggest. Much the most frequent tumor is well-differentiated squamous carcinoma. The tumor cells aggregate as epithelial pearls and there is active keratinisation. Poorly differentiated, anaplastic and small cell tumors are very uncommon.

Carcinoma of the tongue extends locally by penetrating the lingual basement membrane. Tumor cells pass directly to the adjacent soft tissues of the mouth and neck. Lymphatic permeation is early. In the case of the common, anterior tumors, unilateral lymph node metastasis takes place to the submental and submandibular nodes and thence to those of the lower neck. In the case of the rarer, posterior tumors, bilateral spread occurs to the upper cervical nodes. Blood-borne metastasis is commonplace and the deposits are recognized in bone, liver and other sites. The prognosis is poor. Tumors are staged by the TNM system. Those that are unilateral, of small size and without metastasis have a much better outlook than large, posterior tumors with bilateral lymph node involvement. There is a mean 5 -year survival rate of no more than $25 \%$. Treatment is by radical excision followed by irradiation.

\section{Diagnosis}

Physical findings relate to late tumor stage presentation, which is common [1]. Pain in the ear of an adult is a relatively rare problem and usually indicates a malignancy of the oral cavity, oropharynx, or larynx. Formication, the feeling of ants crawling along the lip or cheek, may represent infraorbital nerve invasion by carcinoma. A change in speech is another physical indicator of oral carcinoma. Hoarseness is a sign of vocal cord impairment by local tumor growth. Airway compromise is usually a late symptom but can precipitate an emergency. Evaluation of the patient includes a visualization of the entire upper aerodigestive tract. Careful intraoral examination and indirect mirror laryngoscopy are essential. A flexible nasopharyngoscope has added greatly to complete examination. Mobility of the tongue always should be noted. The position and movement of the vocal cords are also important. Limited motion of the mandible may come from direct tumor invasion or invasion of the tumor through the retromolar trigone. This may cause an uncomfortable symptom of trismus, an ominous clinical sign. Examination of the neck will reveal the presence or absence of metastatic lymph nodes. Careful examination will allow the examiner to assign an $\mathrm{N}$ stage to the patient, which is an important prognostic feature. A careful neurologic examination is also important to reveal evidence of more extensive disease. These findings would include extraocular movement disorders or Horner syndrome from the invasion of cervical sympathetic nerves. Distant metastases are evaluated by laboratory procedures and radiologic examination, as well as by history and physical examination. Pleuritic pain or shortness of breath may indicate lung involvement, and distinct pain at specific sites may indicate bone involvement.

Definitive diagnosis depends on a biopsy. If the primary site is visible, a wedge biopsy should be taken at the edge of the tumor. Because of the significant incidence of synchronous primaries, however, evaluation of the entire upper aerodigestive tract is useful. Triple endoscopy, i.e., bronchoscopy, esophagoscopy, and direct laryngoscopy, is advisable for ideal workup of head and neck cancer. Radiologic evaluation of head and neck disease usually involves 
assessment of the mandible with dental films, mandibular series, and panoramic films. Bone scans frequently are falsely positive; rather, CT scanning is a very sensitive method of diagnosing bone invasion. MRI is probably the most accurate and useful method of evaluating the mandible as well as other areas of head and neck.

\section{Conclusion}

Cancer of the tongue is a type of oral or mouth cancer. It engages the front of the tongue, and cancer on the back of the tongue is called oropharyngeal cancer. This disease most commonly occurs in smokers, in people who frequently consume alcohol, eat little fruits and vegetables, and their diet is predominantly based on red meat, human papillomaviruses, people with a genetic predisposition, and people who have previously had some type of cancer. Cancer of the tongue is regularly painless at an early stage and is usually detected during a routine dental examination. It usually appears on the sides of the tongue. Cancer of the tongue often looks like open fissures and tends to grow into the tissues below. The red area in the mouth is a precursor to cancer. Anyone with a red area on the sides of the tongue should see a doctor or dentist.

\section{References}

[1] Schwartz SI, Shires GT, Spencer FC, Daly JM, Fischer JE, Galloway AC. Principles of Surgery, Companion Handbook, Seventh Edition. McGraw Hill Professional. 1998 Dec 22: 405-18.

[2] Lowry SF, Ciocca RG, Rettie CS, editors. Learning Surgery: The Surgery Clerkship Manual. Springer Science \& Business Media. 2005 Jun 28:191.

[3] Burnand KG, Black J, Corbett SA, Thomas WE, editors. Browse's Introduction to the Symptoms \& Signs of Surgical Disease 4th Edition. CRC Press, Taylor \& Francis Group, Boca Raton, USA. 2005 Jul:258-6o.

[4] Ellis H, Calne R, Watson C. Lecture notes: general surgery 13th Edition. John Wiley \& Sons, Ltd, Chichester, UK. 2016 Feb 26:150.

[5] Gardner D, Tweedle DE, editors. Pathology for Surgeons in Training: An AZ Revision Text, 3rd
Edition. CRC Press, Taylor \& Francis Group, Boca Raton, USA. 2002 Mar 30:258-59. 